\title{
Subretinal Route of Administration
}

National Cancer Institute

\section{Source}

National Cancer Institute. Subretinal Route of Administration. NCI Thesaurus. Code C79143.

Administration beneath the retina. 